Review

\title{
Origin of Terrestrial Bioorganic Homochirality and Symmetry Breaking in the Universe
}

\author{
Jun-ichi Takahashi * and Kensei Kobayashi \\ Faculty of Engineering, Yokohama National University, Yokohama 240-8501, Japan \\ * Correspondence: takahashi-junichi-bd@ynu.ac.jp; Tel.: +81-45-339-3938
}

Received: 5 June 2019; Accepted: 5 July 2019; Published: 15 July 2019

check for updates

\begin{abstract}
The origin of terrestrial bioorganic homochirality is one of the most important and unresolved problems in the study of chemical evolution prior to the origin of terrestrial life. One hypothesis advocated in the context of astrobiology is that polarized quantum radiation in space, such as circularly polarized photons or spin-polarized leptons, induced asymmetric chemical and physical conditions in the primitive interstellar media (the cosmic scenario). Another advocated hypothesis in the context of symmetry breaking in the universe is that the bioorganic asymmetry is intrinsically derived from the chiral asymmetric properties of elementary particles, that is, parity violation in the weak interaction (the intrinsic scenario). In this paper, the features of these two scenarios are discussed and approaches to validate them are reviewed.
\end{abstract}

Keywords: bioorganic homochirality; circularly polarized photon; spin-polarized lepton; parity violation in the weak interaction

\section{Introduction}

A full explanation of the maximally broken symmetry in terrestrial bioorganic homochirality (the enantiomeric domination of L-form amino acids in proteins and D-form sugars in DNA/RNA) has not been sufficiently achieved despite their significance in resolving the problems concerning the origin of terrestrial life. One of the hypotheses for the origin of terrestrial bioorganic homochirality has been advocated to geological points of view based on prebiotic terrestrial circumstances (the terrestrial scenario), such as the preferential adsorption of prebiotic organic molecules on chiral mineral surfaces (e.g., left- and right-handed crystal quartz).

However, extraterrestrial origins of bioorganic homochirality have also been advocated by the researchers, even in the fields of biology and chemistry. One of the most attractive recent hypotheses in the context of astrobiology advocates that polarized quantum radiation in space, such as circularly polarized photons [1-3] or spin-polarized particles, can induce asymmetric conditions in the primitive interstellar media resulting in terrestrial bioorganic homochirality (the cosmic scenario). In particular, nuclear-decay- or cosmic-ray-derived leptons (i.e., electrons, muons, and neutrinos) in nature have a specified helicity, that is, they have a spin angular momentum polarized parallel or antiparallel to their kinetic momentum due to parity violations (PV) in the weak interaction as a result of intrinsic symmetry breaking in nature [4-14].

A second hypothesis has also been advocated in the context of intrinsic symmetry breaking in nature (the intrinsic scenario). This scenario advocates that the bioorganic asymmetry is universally derived from chiral asymmetry breaking at the level of elementary particle interactions, such as PV in the weak interaction, as well as at the level of astrophysics, such as asymmetry in cosmic constructions $[15,16]$. In this case, serious problems related to the considerable discrepancies in the hierarchical structures between the evolution of matter and the chemical evolution of bioorganic compounds needs to be universally resolved. 
From the standpoint of asymmetry in nature, spin-polarized leptons in the above-mentioned cosmic scenario should be included in the intrinsic scenario due to the generation of spin-polarized leptons derived from PV in the weak interaction. In this article, the features of the cosmic and intrinsic scenarios are discussed and experimental research approaches to validate these scenarios are comparatively reviewed. Results of laboratory verification experiments are shown to explain the relationship between bioorganic homochirality and astrophysical asymmetric radiation sources, which are derived from the intrinsic asymmetry of the universe.

\section{Typical Scenarios for the Origin of Homochirality}

\subsection{The Cosmic Scenario}

Because bioorganic compounds synthesized under abiotic circumstances are intrinsically racemic mixtures including equal amounts of $\mathrm{L}-$ and D-bodies, it is hypothesized that asymmetric products originated from "chiral radiation," that is, physically asymmetric excitation sources in space. As a result, "asymmetric seeds" were transported to primitive Earth resulting in terrestrial homochirality via several types of amplification mechanisms (the cosmic Scenario) [1]. One of the most attractive cosmic scenario hypotheses can be summarized in the context of astrobiology as shown below.

(i) Prebiotic simple molecules were densely accumulated on interstellar dust surfaces in dense molecular clouds [17];

(ii) "Chiral radiation" in space, for example, circularly polarized photons or spin-polarized particles, induced asymmetric reactions and produced non-racemic mixtures of chiral complex organic materials including bioorganic precursors as "asymmetric seeds";

(iii) The "asymmetric seeds" were transported with meteorites or asteroids to the primitive Earth resulting in terrestrial bioorganic homochirality via some types of "asymmetric amplification" mechanism.

As evidence supporting this scenario, L-enantiomeric excesses have been observed in isovaline, an $\alpha$-methyl amino acid, and several other $\alpha$-methyl amino acids in the Murchison meteorite [18]. In general, the racemization rate of $\alpha$-methyl amino acids is smaller than that of $\alpha$-hydrogen amino acids. The observed enantiomeric excesses of $\alpha$-methyl amino acids in meteorites suggest that asymmetric reactions on the surface or in the interior of space materials actually occurred and that the consequent enantiomeric excesses were preserved due to their reduced racemization rate. As for "asymmetric amplification" mechanism, the Soai self-autocatalytic reaction with positive feedback amplification can support the almost perfect asymmetry in terrestrial biomolecular homochirality [19].

\subsubsection{Circularly Polarized Photons}

\section{- Observations of circularly polarized radiation in space}

Of the known chiral radiation, circularly polarized photons have been generally considered to be the most typical polarized quantum radiation in space. In fact, high-energy polarized photons have been observed in the X-ray to gamma-ray region. In this high photon energy region, linearly polarized X-rays, which are principally generated by synchrotron radiation (SR) from relativistic kinetic electrons captured by the strong magnetic fields around neutron stars in supernova remnant, have been observed emanating from the Crab Nebula [19-21]. Gamma-ray bursts (GRBs) from supernova explosion areas have been successfully observed with highly linear polarization (up to $70-80 \%$ ) by the ICAROS/GAP satellite project [22]. In addition, faint circularly polarized light ( $0.6 \%$ in fraction) has been observed in the visible region of GRB afterglow, which is supposed to be derived from SR due to anisotropic electron motion in plasma. In general, long bursts are generated by the gravitational collapse of high mass stars and slow bursts are generated by binary neutron star mergers [23].

Recently, polarized photons in near infrared (NIR) regions have also been observed in scattered light from interstellar dust clouds in star formation areas by terrestrial ground-level observatories. 
In particular, increases in both the photon flux and the ratio of radiated circular polarization with increasing star formation area have been reported [24,25]. In the middle infrared (MIR) region, the AKARI satellite has detected a polarization pattern at wavelengths of 3-11 $\mu \mathrm{m}$ from protostars [26].

Circularly and linearly polarized light derived from absorption lines of solar elements due to Zeeman splitting have been observed (anti-Zeeman effects). These effects depend on the angle correlation between the magnetic field and the line of sight. Spectro-polarimetric observations of sunspot magnetic fields have been observed by using near-infrared cameras (e.g., large-format infrared array detectors) [27].

From the far infrared (FIR) to terahertz (THz) wave region, theoretical calculations for the evolution of elliptical galaxies have predicted that, prior to $4 \mathrm{Gyr}$, star formation areas radiated intense FIR to $\mathrm{THz}$ photons and gradually darkened after the star formation events. This prediction means that, in the era of prebiotic molecule formation, FIR to $\mathrm{THz}$ photons were more intense than visible or UV photons. This suggests that polarized photons in the FIR to THz region are also important candidates for "chiral radiation."

In contrast to the above-mentioned area- and time-localized radiation sources in space, cosmic microwave background radiation is thought to be equally distributed in space; however, localized polarization patterns spreading in a specific direction (B-mode/E-mode polarization) have recently been observed. The direct relationship between polarized microwave radiation and chiral asymmetric reactions in bioorganic molecules remains uncertain. The observed polarization is likely relevance to the non-uniform structure of space resulting in the above-mentioned generation of the polarized radiation sources in space [28].

- Chiral reaction scheme via circularly polarized photons

Asymmetric reaction schemes due to circularly polarized photons can be classified corresponding to the photon energy region. Gamma-rays (the photon-nuclear reaction physics region) indicate nuclear excitation with an intense electromagnetic field. X-rays (the intermediate region between radiation chemistry and photochemistry) indicate core electron excitation to optical-active excited states via single photon absorption. VUV-UV-VIS (the photochemistry region) indicates valence electron excitation to optical-active excited states via single photon absorption. NIR-MIR (the intermediate region between photochemistry and thermochemistry) indicates vibrational or rotational excitation of molecular bonds to optical-active excited states. FIR-THz-MW (radiochemistry region) indicates perturbation to intermolecular interactions via multi-photon absorption.

- Circular dichroism

Interactions of circularly polarized photons with chiral molecules can be estimated by using circular dichroism (CD), which is defined as the photo-absorption difference between left- and right-circularly polarized photons at specific wavelengths. Chiral bioorganic molecules generally have intense $C D$ chromophores derived from characteristic electronic transitions corresponding to photon energies in the visible to UV region. Because the electronic transitions related to $\mathrm{CD}$ chromophores reflect the bond structures and base conformations of molecules, CD spectra sensitively reflect the steric structures of chiral molecules with a high degree of accuracy $[29,30]$. From this point of view, it is suggested that the expected characteristics of asymmetric photochemical reactions strongly depend on the irradiated circularly polarized light (CPL) wavelength. For example, theoretical calculations of CD spectrum of alanine molecules have revealed that the chromophores derived from electronic transitions correspond to several different wavelengths in the $120-230 \mathrm{~nm}$ region [31]. Chiral molecules also have $\mathrm{CD}$ chromophores derived from characteristic vibrational and rotational transitions corresponding to photon energies in the IR region. In terahertz wave region, absorption spectra for chiral molecule crystals can discriminate between chiral crystals (L-alanine and D-alanine) and achiral crystals (DL-alanine) because terahertz region spectra reflect minute intermolecular interactions [31]. 


\subsubsection{Spin-Polarized Leptons}

Another type of polarized source that is consistent with the proposed cosmic scenario hypothesis is spin-polarized leptons, i.e., electrons/positrons, muons, and neutrinos, produced in space. Leptons are classified as basic elementary particles, constructed in the standard model of particle physics via quarks, gauge bosons, and Higgs bosons. Lepton and quark interactions are dominated by the parity-violating weak interaction.

\section{- Electrons/Positrons}

Of the leptons, electrons are one of the most universally present particles in ordinary materials. Spin-polarized electrons in nature are emitted with $\beta^{-}$-decay from radioactive nuclear particles derived from PV involving the weak nuclear interaction $\left(\mathrm{n} \rightarrow \mathrm{p}+\mathrm{e}^{-}+\bar{v}_{\mathrm{e}}\right)$ and spin-polarized positrons (the anti-particle of electrons) from $\beta^{+}$-decay $\left(\mathrm{p} \rightarrow \mathrm{n}+\mathrm{e}^{+}+v_{\mathrm{e}}\right)$. In $\beta^{-} / \beta^{+}$-decay, with the weak interaction, the spin angular momentum vectors of electron/positron are perfectly polarized as antiparallel/parallel to the vector direction of the kinetic momentum. In this meaning, spin-polarized electrons/positrons are "chiral radiation" as well as muons and neutrinos which will be mentioned at the paragraphs below. It is expected that the spin-polarized leptons will induce the different type of chemical reactions form CPL induced reactions.

Radiation of spin-polarized electrons and positrons are likely frequent events from the nuclear $\beta^{-} / \beta^{+}$-decay of radioactive nuclei in space. In particular, chiral chemical reactions should be induced by negative helicity electrons in $\beta^{-}$-decay from radioactive nuclei such as ${ }^{60} \mathrm{Co}$ and ${ }^{90} \mathrm{Sr}$ in space [4-9]. It has been well known that $\beta^{-} / \beta^{+}$-decays heat of ${ }^{60} \mathrm{Fe}$ and ${ }^{26} \mathrm{Al}$ in meteorite parent body can operate as a heat source for the formation and differentiation of its organic materials. From the standpoint of astrochemistry, spin-polarized electrons in $\beta^{-} / \beta^{+}$-decay potentially operate as chiral radiation to induce chiral reaction processes in organic materials in the meteorites.

Interaction schemes of spin-polarized positrons with chiral molecules should be recognizable as being very different from those of electrons. It has been suggested that the electron-positron annihilation phenomena induced by spin-polarized positrons present a dependence on the spin distribution conditions of the target bioorganic molecules.

Accompanied by electron/positron radiation in $\beta^{-} / \beta^{+}$-decay from radioactive nuclei, gamma-rays can be emitted from the disintegrated nuclei via the electromagnetic transition from excited states to lower-level states. The polarization of the emitted gamma-rays depends on the conservation lows of the spin and orbital angular momentums between the nuclei, electron/positron, anti-neutrino/neutrino, and gamma-photon, which is limited according to conservation laws by PV in the weak interaction. For example, $\beta^{-}$-decay from ${ }^{60} \mathrm{Co}$ is accompanied by circularly polarized gamma-rays whereas $\beta^{-}$ decay from ${ }^{90} \mathrm{Sr}$ is not accompanied by any gamma-rays due to its direct transition to the ground state of the disintegrated nucleus.

\section{- Muons}

In the atmosphere of the Earth, muons are generated as a secondary cosmic ray via pion-decay following the impact of a cosmic ray proton with an atmospheric atom. The spin angular momentum of positive $\left(\mu^{+}\right)$and negative $\left(\mu^{-}\right)$muons in nature is intrinsically polarized with negative and positive helicity (left- and right-handed polarization), respectively, by PV in the weak interaction. Muons are lepton particles with the same value of negative $\left(\mu^{-}\right)$or positive $\left(\mu^{+}\right)$charge as electrons or positrons, respectively, and the same value of spin $(1 / 2)$ as electrons and positrons. Because the muon mass is much larger than the electron mass $\left(\mathrm{m}_{\mu} / \mathrm{m}_{\mathrm{e}} \sim 200\right)$ and close to the proton mass $\left(\mathrm{m}_{\mu} / \mathrm{m}_{\mathrm{p}} \sim 1 / 9\right)$, interactions of spin-polarized muons with organic molecules, including asymmetric reactions, are different from those of spin-polarized electrons or positrons.

In the positive muon irradiation case, muonium (a positive muon-electron pair $(\mathrm{Mu})$ ) and a non-bonding lone electron can be formed on a double-bond of an organic molecule resulting in the formation of "Mu radicals." As a result, radical-mediated asymmetric reactions can be expected in 
organic molecules. Double bonds of carbon-carbon can be reduced to a single bond resulting in rotation around the single-bond axis. In addition, hot Mu generated by a muon beam can induce hydrogen extraction from organic molecule $\left(\mathrm{Mu}^{*}+\mathrm{RH} \rightarrow \mathrm{MuH}+\mathrm{R}\right)$. Via these types of reactions, racemization, that is, the enantiomer type conversion of chiral molecules) such as amino acids, is strongly expected. In addition, Mu can attack non-bonding electrons of the carboxyl bases of amino acids, resulting in asymmetric electronic excitation depending on the direction of spin polarization of the muon irradiation via several types of processes. Mu can be thought of a type of isotope of the $\mathrm{H}$ atom whose atomic total mass is $1 / 9$ that of hydrogen. Conversely, the reduced mass of $\mathrm{Mu}$ (positive muon-electron) and an $\mathrm{H}$ atom (proton-electron) with a $1 \mathrm{~s}$ orbital electron are approximately equivalent values. From the standpoint of the electronic state, $\mathrm{Mu}$ and an $\mathrm{H}$ atom can be regarded as being chemically equivalent.

Interactions of positive and negative muons with organic molecules are very different from each other due to the relatively heavy mass of a muon. In the negative muon beam case, a negative muon combined with an atomic nucleus makes a muonic atom (atomic nucleus-negative muons pair). Muonic atom formation can induce a charge shielding effect due to heavy negative muons captured in small orbit radii, resulting in the formation of apparently $Z-1$ nucleus and negative ion. Further, atomic conversion $(Z \rightarrow Z-1)$ via $\beta$-decay $\left(\mu^{-}+p \rightarrow n+\gamma_{\mu}\right)$ can occur in large atomic number nucleus. It is also expected that the chemical activity of muonic atoms depends on the direction of spin polarization of the muon irradiation.

From these viewpoints, spin-polarized muon irradiation has the potential to induce novel types of optical activities different from those of polarized photon and spin-polarized electron irradiation.

\section{- Neutrinos}

In general, the interaction of neutrinos with materials is very weak on the Earth. However, reactions including neutrinos accompanied by the weak interaction cannot be neglected in high density nuclear materials $\left(>10^{11} \mathrm{~g} / \mathrm{cm}^{3}\right)$ in space.

As for high-energy astrophysical objects, neutrons released in supernova explosions can cause chiral asymmetry in molecules in interstellar gas-dust clouds. The specific physical mechanism of a relativistic neutron fireball, which is a relativistic chiral electron-proton plasma, has been advocated, in which the electrons carrying their helicity to the cloud show high chiral efficiency $[5,6,9]$.

Neutrinos with a specified spin angular momentum due to the PV weak interaction are also radiated in supernova explosions. Recently, it has been argued that selective destruction of one amino acid chirality by electron anti-neutrinos and the magnetic field from a supernova resulted in the production of meteorites including left-handed amino acids [11-14]. In this hypothesis, spin-spin interactions between neutrinos and nitrogen nuclei are emphasized.

\subsection{The Intrinsic Scenario}

Another hypothesis has been advocated in the context of intrinsic symmetry breaking in nature, that is, the bioorganic asymmetry is universally derived from chiral asymmetry breaking at the level of elementary particles, such as PV in the weak interaction [15]. It has been argued that PV leads to energy differences in enantiomers in the $10^{-12}$ joule/mole range and that theoretical calculations of the energy differences in enantiomers indicate that the intrinsic energies of $\mathrm{L}$-amino acids and D-ribose are slightly lower than those of their corresponding enantiomers (D-amino acids and L-ribose). Quack considered that electroweak quantum chemistry methods predict such energy differences to be one to two orders of magnitude larger than previously accepted, but still very small, and discussed the current status of theory and some current experimental approaches [16]. 


\section{Experimental Approaches to Examine the Scenarios}

\subsection{The Cosmic Scenario}

\subsubsection{Circularly Polarized Photons}

The first asymmetric photolysis experiment on a racemic organic molecule (2-bromopropanoic acid ethyl ester) using CPL was reported by Kuhn [32] in 1929. In the 1970's, several asymmetric photolysis experiments were conducted on racemic molecules, i.e., metal oxalate ions [33] and amino acid ((RS)-leucine) [34], using CPL from high-pressure mercury lamps.

After the 1990's, SR facilities were used as light sources for photochemical reactions, spectroscopic analyses, and micro-fabrication. In particular, the asymmetric photolysis of racemic amino acids in an aqueous solution using CPL from SR was reported as a validation of the "cosmic scenario" in the context of astrobiology. Takano et al. reported the asymmetric photolysis of racemic isovaline [35], and Nishino et al. reported the $\mathrm{pH}$ dependence of leucine asymmetric photolysis [36]. In these experiments, they were able to selectively photolyze one optical isomer in an optically active compound depending on its CD in the wavelength region of the irradiated CPL.

Further, Takano et al. reported the CPL irradiation of an aqueous solution of complex organic compounds with high molecular weight, which involved amino-acid precursors with molecular weights of several thousands. After CPL irradiation followed by the acid-hydrolysis of the solution, positive and negative enantiomeric excesses in alanine were successfully detected [37,38].

Conversely, in molecular clouds, organic molecules on interstellar dust surfaces should be in their solid phase, for example, the condensed ice mantle around silica dust core (Greenberg Model [17]). From this viewpoint, solid-phase experiments can contribute to verifying reaction models of on-surface or surface-catalytic reactions on materials such as interstellar dust. Meierhenrich et al. [39] reported solid-phase leucine photolysis and Takahashi reported solid-phase phenylalanine photolysis using polarized SR in the VUV region [40].

Recent verification experiments have been performed to examine the relationship between optical activity emergence into several target materials and asymmetric radiation, typically CPL. We irradiated racemic D L -amino acid films with monochromatic CPL with a wavelength of $215 \mathrm{~nm}$ from the free electron laser (FEL) of UVSOR-II at Institute for Molecular Science (IMS), Okazaki, Japan [31]. This result suggest that the racemic mixture of chiral amino acid was differentially photolyzed or transformed depending on the CD at the irradiated CPL wavelength.

We are using CD spectroscopy to evaluate optical activity emergence because the CD spectrum sensitively reflects the steric structures of chiral molecules with a high degree of accuracy. Theoretical calculations of the $\mathrm{CD}$ spectrum of the alanine molecule have revealed that the circular dichroism chromophores derived from characteristic electronic transitions $\left(n-\pi^{*}, \pi-\pi^{*}\right.$ for the carboxyl group and $\mathrm{n}-\sigma^{*}, \pi-\sigma^{*}$ for the amino group) correspond to several different wavelengths in the $120-230 \mathrm{~nm}$ region [30]. From this standpoint, the expected characteristics of optical activity emergence strongly depend on the irradiated CPL wavelength. In further experiments to validate the scenario, we are investigating the dependence on the irradiation wavelength using CPL with wavelengths of $230 \mathrm{~nm}$, $203 \mathrm{~nm}, 180 \mathrm{~nm}$, and $155 \mathrm{~nm}$ from the undulator beam line BL1U of UVSOR-III at IMS. To examine optical activity emergence, we measured the $C D$ and photo absorption spectra of the deposited films using a commercial CD spectrometer (JASCO J-725) and the SR CD beam line BL-12 of Hiroshima Synchrotron Radiation Center. A detailed analysis of the CD spectra is in progress to determine the complete mechanism for optical activity emergence [41,42].

Similar irradiation experiments for solid-phase films of an intrinsically optically non-active achiral molecule, hydantoin (glycine precursor molecules), and glycine have also been performed using CPL with a wavelength of $215 \mathrm{~nm}$ from FEL of UVSOR-II and BL1U of UVSOR-III, respectively [43]. The CD spectra of irradiated hydantoin and glycine films have many common characteristics, that is, clear peaks newly appear in the CD spectra at $215 \mathrm{~nm}$ and $180 \mathrm{~nm}$ and nearly completely symmetric spectra 
were observed with L- and R-CPL irradiation. Further, the intensity ratio of the two peaks and the intensity and sign of the $\mathrm{CD}$ peaks changed with the irradiation dose. These results suggest that several new chiral structures were introduced into the achiral molecule film by the CPL irradiation. The absorbance of hydantoin after the CPL irradiation increased on the low-energy side but decreased on the high-energy side, suggesting that a molecular structure change occurred in the film.

As for the hydantoin case, first principal simulation calculations of the asymmetric optical response of hydantoin have also been performed. The calculation results suggest that some types of chiral structures derived from a distortion of the five-ring construction were introduced into the racemic film by the CPL irradiation. Analogous to hydantoin, it is suggested that some types of chiral structures were introduced into the racemic glycine film by the CPL irradiation [44]. It is known that crystalized glycine contains several crystal polymorphs including left- or right-handed spiral constructions, similar to crystalline quartz. The experimental results suggest that the CPL irradiation induced construction distortion including left- or right-handed spiral crystal polymorphs depending on the irradiated CPL chirality. Similar types of mechanisms including chiral structure formation are potentially applicable to racemic mixtures of optically active amino acids (i.e., DL-alanine), which is potentially relevant to the origin of the terrestrial bioorganic homochirality stimulated by "chiral radiation."

In addition to solid films of amino acids, ribose and related sugars following ultraviolet irradiation of interstellar ice fixed in ice analogs have also been reported with high-resolution mass spectroscopy [44-46].

\subsubsection{Spin-Polarized Leptons}

- Electrons/Positrons

The first pioneering experiment examining asymmetric radical formation in D- and L-alanines using $\beta^{-}$-irradiation was conducted by Akaboshi, et al. [47]. Tsarev et al. reported that, after amino acid/metal complexes were irradiated with high flux $\beta^{-}$-rays from an ${ }^{90} \mathrm{Sr}^{90}{ }^{90}$ source $(50 \mathrm{Ci})$, at the Russian Federal Nuclear Center in Snezhinsk, and small amounts of enantiomeric excesses were detected in the irradiated products using CD spectroscopy [7-9]. As for interactions of spin-polarized electrons with chiral molecules, Kessler, et al. reported on a low energy $(<10 \mathrm{eV})$ spin-polarized electron beam with a chiral bromocamphor molecule, that is, so-called "spin-polarization dichroism" [48]. Recently, Rosenberg et al. reported interactions of spin-polarized X-ray photoelectron emitted from solid surfaces with chiral molecules adsorbed on them [49,50].

We also recently carried out the same irradiation experiments with amino-acid precursors or solid films of amino acids and also detected enantiomeric excesses using CD spectroscopy on an irradiated isovaline film [51]. Because the helicity of the spin-polarized electron beam in a $\beta^{-}$-ray is negative due to $P V$ in the weak interaction, verification experiments are required using a spin-polarized electron beam with positive helicity to detect enantiomeric excesses with the opposite sign with respect to the negative helicity case. Our present research focuses on simulated experiments using a spin-polarized electron beam with a helicity-controlled irradiating amino acid film in the achiral state (optically non-active) and observing the emergence of optical activity.

\section{- Muons}

No significant difference has been reported for the asymmetric reactions in enantiomers of organic compounds using muon beams, except that Lemmon et al. who reported reactions of spin-polarized positive muons with alanines and octanols [52]. We conducted a trial of spin-polarized muons to examine the optical activity of organic compounds such as amino acids and their precursor molecule in achiral states. In the experiments, a racemic amino acid (DL-alanine) and a precursor molecule of glycine (hydantoin) were irradiated by positive muons with negative helicity and negative muons with positive helicity using the Muon S beam line of the Materials and Life Science Experimental Facility (MLF) in the Japan Proton Accelerator Research Complex (J-PARC). Because the penetrating depth of the muon beam through the polyethylene pellet is estimated to be less than $1 \mathrm{~mm}$, nearly all the muon 
beam should be captured with the target materials packed in the polyethylene or potassium bromide circular cylinder pellet. Optical activity measurements of the irradiated samples are in progress. We expect to reveal a mechanism for optical activity emergence whose sign depends on the direction of the spin polarization and the charge of the muon. We are also planning to observe the disturbance of the muon spin polarization after chiral chemical reactions due to muonium or muonic atom formation in the target chiral molecules. The disturbance of the muon spin polarization can be observed using measurements of the distribution of positrons decayed from muons.

\subsection{The Intrinsic Scenario}

Recently, the first set of observations of PV energy differences between two enantiomers of chiral molecules was presented using high-resolution laser spectroscopy [53]. However, at present, no successful experiments have been reported. The role of PV in the weak interaction in the origin of terrestrial homochirality is a subject that cuts across research fields, including fundamental physics, nuclear cosmology, theoretical chemistry, and quantum spectroscopy.

\section{Conclusions}

Various scenarios for the origin of the homochirality of terrestrial bioorganic molecules in the context of astrobiology (the cosmic scenario) have been proposed. Asymmetric radiation in space, such as circularly polarized photons or spin-polarized leptons, induced asymmetric conditions in the primitive interstellar media. We are conducting cooperative investigations using the following observations, experiments and computational calculations to examine the cosmic scenario $[54,55]$.

(1) Astronomical observations of polarized radiation from various star-forming and high-energy phenomena burst regions in space using highly sensitive polarization detecting systems settled in astronomical observatories;

(2) Experiments with polarized quantum beams from high-energy particle accelerators irradiating amino acids or sugars and their precursor molecules, followed by chemical and optical measurements of enantiomeric excesses;

(3) First principal calculations of asymmetric optical responses and the subsequent asymmetric chemical reactions for amino acids or sugars and their precursor molecules, including the intrinsic energy difference between the enantiomers derived from PV.

The synergetic use of these three approaches is expected to help solve the problems of the origin of terrestrial biological homochirality.

Funding: This research was funded by Astrobiology Center, National Institutes of Natural Sciences (NINS-ABC) Project Research-2015 and -2017.

Acknowledgments: The author would like to thank Vladimir A. Tsarev, Takeshi Saito, Kazumichi Nakagawa, Uwe J. Meierhenrich, Masahiro Katoh, Motohide Tamura, Masayuki Umemura, Yoshinori Takano, Koichi Matsuo, Yudai Izumi, Masateru Fujimoto, Yoko Kebukawa, Hiromi Shibata, M. Kenya Kubo, Yasuhiro Miyake and Michiya Fujiki for their collaborations and useful discussions.

Conflicts of Interest: The authors declare no conflicts of interest.

\section{References}

1. Bonner, W.A. The origin and amplification of bioorganic chirality. Orig. Life Evol. Biosph. 1991, $21,59-111$. [CrossRef] [PubMed]

2. Bailey, J.; Chrysostomou, A.; Hough, J.; Gledhill, T.; McCall, A.; Clark, S.; Menard, F.; Tamura, M. Circular polarization in star-formation regions: Implications for bioorganic homochirality. Science 1998, 281, 672-674. [CrossRef] [PubMed]

3. Meierhenrich, U.J. Amino acids and the Asymmetry of Life. In Advances in Astrobiology and Biogeophysics; Brack, A., Horneck, G., McKay, C.P., Stan-Lotter, H., Eds.; Springer: Berlin/Heidelberg, Germany, 2008. 
4. Garay, A.S.; Keszthelyi, L.; Demeter, I.; Hrasko, P. Origin of asymmetry in biomolecules. Nature 1974, 250, 332-333. [CrossRef] [PubMed]

5. Cline, D.B.; Liu, Y.; Wang, H. Effect of a chiral impulse on the weak interaction induced handedness in a prebiotic medium. Orig. Life Evol. Biosph. 1995, 25, 201-209. [CrossRef] [PubMed]

6. Cline, D.B. Supernova antineutrino interactions cause chiral symmetry breaking and possibly homochiral biomaterials for life. Chirality 2005, 17, S234-S239. [CrossRef] [PubMed]

7. Gusev, G.A.; Saito, T.; Tsarev, V.A.; Uryson, A.V. A relativistic neutron fireball from a supernova explosion as a possible source of chiral influence. Orig. Life Evol. Biosph. 2007, 37, 259-266. [CrossRef] [PubMed]

8. Gusev, G.A.; Kobayashi, K.; Moiseenko, E.V.; Poluhina, N.G.; Saito, T.; Ye, T.; Tsarev, V.A.; Xu, J.; Huang, Y.; Zhang, G. Results of the second stage of the investigation of the radiation mechanism of chiral influence (RAMBAS-2 experiment). Orig. Life Evol. Biosph. 2008, 38, 509-515. [CrossRef] [PubMed]

9. Tsarev, V.A. Physical and astrophysical aspects of the problem of origin of chiral asymmetry of the biosphere. Phys. Part Nucl. 2009, 40, 998-1029. [CrossRef]

10. Cline, D.B. Possible physical mechanisms in the galaxy to cause homochiral biomaterials for life. Symmetry 2010, 2, 1450-1460. [CrossRef]

11. Boyd, R.N.; Kajino, T.; Onaka, T. Supernovae and the chirality of the amino acids. Astrobiology 2010, 10, 561-568. [CrossRef]

12. Boyd, R.N.; Kajino, T.; Onaka, T. Supernovae, neutrinos, and the chirality of the amino acids. Int. J. Mol. Sci. 2011, 12, 3432-3444. [CrossRef] [PubMed]

13. Famiano, M.; Boyd, R.N.; Kajino, T.; Onaka, T.; Koehler, K.; Hulbert, S. Determining amino acid chirality in the supernova neutrino processing model. Symmetry 2014, 6, 909-925. [CrossRef]

14. Famiano, M.; Boyd, R.N.; Kajino, T.; Onaka, T. Selection of amino acid chirality via neutrino interactions with ${ }^{14} \mathrm{~N}$ in crossed electric and magnetic fields. Astrobiology 2018, 18, 190-206. [CrossRef] [PubMed]

15. Mason, S.F. Origin of bioorganic handedness. Nature 1984, 311, 19-23. [CrossRef] [PubMed]

16. Quack, M. How important is parity violation for molecular and bioorganic chirality? Angew. Chem. Int. Ed. 2002, 41, 4618-4630. [CrossRef] [PubMed]

17. Greenberg, J.M.; Kouchi, A.; Niesson, W.; Irth, H.; van Paradijs, J.; de Groot, M.; Hermsen, W. Interstellar dust, chirality, comets, and the origins of life: Life from dead stars? J. Biol. Phys. 1994, 20, 61-70. [CrossRef]

18. Cronin, J.R.; Pizzarello, S. Enantiomeric excesses in meteoritic amino acids. Science 1997, 275, 951-955. [CrossRef]

19. Soai, K.; Kawasaki, T. Asymmetric Autocalysis with Amplification of Chirality. Top. Curr. Chem. 2008, 284, $1-33$.

20. Chauvin, M.; Florén, H.-G.; Jackson, M.; Kamae, T.; Kawano, T.; Kiss, M.; Kole, M.; Mikhalev, V.; Moretti, E.; Olofsson, G.; et al. Observation of polarized hard X-ray emission from the Crab by the PoGOLite Pathfinder. Month. Not. R. Astron. Soc. Lett. 2016, 456, L84-L88. [CrossRef]

21. Chauvin, M.; Florén, H.-G.; Friis, M.; Jackson, M.; Kamae, T.; Kataoka, J.; Kawano, T.; Kiss, M.; Mikhalev, V.; Mizuno, T.; et al. Shedding new light on the Crab with polarized X-rays. Sci. Rep. 2017, 7, 7816. [CrossRef]

22. Yonetoku, D.; Murakami, T.; Sakashita, T.; Morihara, Y.; Kikuchi, Y.; Takahashi, T.; Gunji, S.; Mihara, T.; Kubo, S. Detection of Gamma-Ray Polarization in Prompt Emission of GRB 100826A. Astrophys. J. 2011, 743, L30. [CrossRef]

23. Wiersema, K.; Covino, S.; Toma, K.; van der Horst, A.J.; Varela, K.; Min, M.; Greiner, J.; Starling, R.L.C.; Tanvir, N.R.; Wijers, R.A.M.J.; et al. Circular polarization in the optical afterglow of GRB 121024A. Nature 2014, 509, 201-204. [CrossRef] [PubMed]

24. Fukue, T.; Tamura, M.; Kandori, R.; Kusakabe, N.; Hough, J.H.; Lucas, P.W.; Bailey, J.; Whittet, D.C.B.; Nakajima, Y.; Hashimoto, J.; et al. Near-infrared circular polarimetry and correlation diagrams in the Orion Becklin-Neugebauer/Kleinman-Low region: Contribution of dichroic extinction. Astrophys. J. Lett. 2009, 692, L88-L91. [CrossRef]

25. Kwon, J.; Tamura, M.; Lucas, P.W.; Hashimoto, J.; Kusakabe, N.; Kandori, R.; Nakajima, Y.; Nagayama, T.; Nagata, T.; Hough, J.H. Near infrared circular polarization images of NGC 6334-V. Astrophys. J. Lett. 2013, 765, L6. [CrossRef]

26. Neha, S.; Maheswar, G.; Soam, A.; Lee, C.W. Polarization of seven MBM clouds at high galactic latitude. Month. Not. R. Astron. Soc. 2018, 476, 4442-4458. [CrossRef] 
27. Sakurai, T.; Yanagisawa, K.; Kobiki, T.; Kasahara, S.; Nakakubo, K. Sunspot magnetic fields observed with a large-format infrared array. Publ. Astron. Soc. Jpn. 2001, 53, 923-930. [CrossRef]

28. Barkats, D.; Bischoff, C.; Farese, P.; Fitzpatrick, L.; Gaier, T.; Gundersen, J.O.; Hedman, M.M.; Hyatt, L.; Mcmahon, J.J.; Samtleben, D.; et al. First measurements of the polarization of the cosmic microwave background radiation at small angular scales from CAPMAP. Astrophys. J. 2005, 619, L127-L130. [CrossRef]

29. Matsuo, K.; Matsushima, Y.; Fukuyama, T.; Senba, S.; Gekko, K. Vacuum-ultravilet circulardichroism of amino acids as revealed by synchrotron radiation spectrophotometer. Chem. Lett. 2002, 31, 826-827. [CrossRef]

30. Tanaka, M.; Kodama, Y.; Nakagawa, K. Circular dichroism of amino acid films in UV-VUV region. Enantiomer 2002, 7, 185-190. [CrossRef]

31. Kaneko, F.; Yagi-Watanabe, K.; Tanaka, M.; Nakagawa, K. Natural circular dichroism spectra of alanine and valine films in vacuum ultraviolet region. J. Phys. Soc. Jpn. 2009, 78, 013001. [CrossRef]

32. Kuhn, W.; Braun, E. Photochemische erzeugung optisch aktiver stoffe. Naturwissenschaften 1929, 17, $227-228$. [CrossRef]

33. Norden, B. Optical activity developed by preferential racemization of one enantiomer in Racemic Cr(III) $(\mathrm{ox})_{3}{ }^{3-}$ induced by irradiation with circularly polarized light. Acta Chem. Scand. 1970, 24, 349-351. [CrossRef]

34. Flores, J.J.; Bonner, W.A.; Massey, G.A. Asymmetric photolysis of (RS)-leucine with circularly polarized ultraviolet light. J. Am. Chem. Soc. 1977, 99, 3622-3625. [CrossRef] [PubMed]

35. Takano, Y.; Kaneko, T.; Kobayashi, K.; Takahashi, J. Asymmetric photolysis of (DL)-isovaline by synchrotron radiation. Orig. Life Evol. Biosph. 2002, 32, 447-448.

36. Nishino, H.; Kosaka, A.; Hembury, G.A.; Shitomi, H.; Onuki, H.; Inoue, Y. Mechanism of pH dependent photolysis of aliphatic amino acids and enantiomeric enrichment of racemic leucine by circularly polarized light. Org. Lett. 2001, 3, 921-924. [CrossRef] [PubMed]

37. Takano, Y.; Takahashi, J.; Kaneko, T.; Marumo, K.; Kobayashi, K. Asymmetric synthesis of amino acid precursors in interstellar complex organics by circularly polarized light. Earth Planet. Sci. Lett. 2007, 254, 106-114. [CrossRef]

38. Kobayashi, K.; Kaneko, K.; Takahashi, J.; Takano, Y. High molecular weight complex organics in interstellar space and their relevance to origins of life. In Astrobiology: From Simple Molecules to Primitive Life; Basiuk, V., Ed.; American Scientific Publisher: Valencia, CA, USA, 2008.

39. Meierhenrich, U.J.; Nahon, L.; Alcaraz, C.; Bredehöft, J.H.; Hoffmann, S.V.; Barbier, B.; Brack, A. Asymmetric vacuum UV photolysis of the amino acid leucine in the solid state. Angew. Chem. Int. Ed. 2005, 44, 5630-5634. [CrossRef] [PubMed]

40. Takahashi, J. Asymmetric photolysis of thin solid film of aromatic amino acid with circularly polarized light. Orig. Life Evol. Biosph. 2006, 36, 280-282.

41. Takahashi, J.; Shinojima, H.; Seyama, M.; Ueno, Y.; Kaneko, T.; Kobayashi, K.; Mita, H.; Adachi, M.; Hosaka, M.; Katoh, M. Chirality emergence in thin solid fFilms of amino acids by polarized light from synchrotron radiation and free electron laser. Int. J. Mol. Sci. 2009, 10, 3044-3064. [CrossRef]

42. Matsuo, K.; Izumi, Y.; Takahashi, J.; Fujimoto, M.; Katoh, M. Emergence of biological homochirality by irradiation of polarized quantum beams. UVSOR Act. Rep. 2016 2017, 44, 157.

43. Takahashi, J.; Sakamoto, T.; Izumi, Y.; Matsuo, K.; Fujimoto, M.; Katoh, M.; Kebukawa, Y.; Kobayashi, K. Circular dichroism analysis of optical activity emergence in amino-acid thin films irradiated by vacuum-ultraviolet circularly-polarized light. In Proceedings of the 22nd Hiroshima International Symposium on Synchrotron Radiation, Higashi-Hiroshima, Japan, 7-8 March 2019.

44. Takahashi, J.; Suzuki, N.; Kebukawa, Y.; Kobayashi, K.; Izumi, Y.; Matsuo, K.; Fujimoto, M.; Katoh, M. Optical activity emergence in glycine by circularly polarized light. UVSOR Act. Rep. 2016 2017, 44, 156.

45. Meinert, C.; Myrgorodska, I.; Marcellus, P.; Buhse, T.; Nahon, L.; Hoffmann, S.V.; d'Hendecourt, L.L.S.; Meierhenrich, U.J. Ribose and related sugars from ultraviolet irradiation of interstellar ice analogs. Science 2016, 352, 208-212. [CrossRef] [PubMed]

46. Garcia, A.D.; Meinert, C.; Sugahara, H.; Jones, N.C.; Hoffmann, S.V.; Meierhenrich, U.J. The Astrophysical Formation of Asymmetric Molecules and the Emergence of a Chiral Bias. Life 2019, 9, 29. [CrossRef] [PubMed]

47. Akaboshi, M.; Noda, M.; Kawai, K.; Maki, H.; Kawamoto, K. Asymmetrical radical formation in D- and L-alanines irradiated with yttrium-90ß-rays. Orig. Life 1979, 9, 181-186. [CrossRef] [PubMed] 
48. Kessler, J. Electron Dichroism: Interaction of Polarized Electrons with Chiral Molecules. Phys. Essays 2000, 13, 421-426. [CrossRef]

49. Rosenberg, R.A.; Abu Haija, M.; Ryan, P.J. Chiral-selective chemistry induced by spin-polarized secondary electrons from a magnetic substrate. Phys. Rev. Lett. 2008, 101, 178301. [CrossRef]

50. Rosenberg, R.A.; Mishra, D.; Naaman, R. Chiral selective chemistry induced by natural selection of spin-solarized electrons. Angew. Chem. Int. Ed. 2015, 54, 7295-7298. [CrossRef]

51. Burkov, V.I.; Goncharova, L.A.; Gusev, G.A.; Hashimoto, H.; Kaneko, F.; Kaneko, T.; Kobayashi, K.; Mita, H.; Moiseenko, E.V.; Ogawa, T.; et al. Asymmetric reactions of amino-acid-related compounds by polarized electrons from beta-decay radiation. Orig. Life Evol. Biosph. 2009, 39, 295-296.

52. Lemmon, R.M.; Crowe, K.M.; Gygax, F.N.; Johnson, R.F.; Patterson, B.D.; Brewer, J.H.; Fleming, D.G. Search for selectivity between optical isomers in reactions of polarized positive muons with alanines and octanols. Nature 1974, 252, 692-694. [CrossRef]

53. Darquié, B.; Stoeffler, C.; Shelkovnikov, A.; Daussy, C.; Amy-Klein, A.; Chardonnet, C.; Zrig, S.; Guy, L.; Crassous, J.; Soulard, P.; et al. Progress toward a first observation of parity violation in chiral molecules by high-resolution laser spectroscopy. Chirality 2010, 22, 870-884. [CrossRef]

54. Takahashi, J.; Katoh, M.; Tamura, M.; Umemura, M.; Kusakabe, N.; Kwon, J.; Kobayashi, K.; Takashima, Y.; Hosaka, M.; Zen, H.; et al. NINS Astrobiology Center project: The origin of terrestrial bioorganic homochirality relevance to asymmetry of the universe-Approaches with synergy effects of observations, experiments and computations. In Proceedings of the 26th Goldschmidt Conference (Goldschmidt2016), Yokohama, Japan, 26 June-1 July 2016.

55. Takahashi, J. Biological homochirality and symmertry breaking of the universe. In Proceedings of the XVIII International Conference on the Origin of Life (ISSOL2017), San Diego, CA, USA, 16-21 July 2017.

(C) 2019 by the authors. Licensee MDPI, Basel, Switzerland. This article is an open access article distributed under the terms and conditions of the Creative Commons Attribution (CC BY) license (http://creativecommons.org/licenses/by/4.0/). 